\title{
ISOPARAMETRIC SUBMANIFOLDS OF HYPERBOLIC SPACES
}

\author{
BINGLE WU
}

\begin{abstract}
In this paper we prove a decomposition theorem for isoparametric submanifolds of hyperbolic spaces. And as a consequence we obtain all polar actions on hyperbolic spaces. We also prove that any isoparametric submanifold of infinite dimensional hyperbolic space is either totally geodesic, or finite dimensional.
\end{abstract}

\section{INTRODUCTION}

In the late 1930s Élie Cartan defined the notion of isoparametric hypersurface of a space form, and he proved that an isoparametric hypersurface of Euclidean space is a totally umbilic hypersurface; an isoparametric hypersurface of a hyperbolic space is either a totally umbilic hypersurface, or the standard product $S^{k} \times H^{n-k}$ in $H^{n+1}$. But for the sphere case, isoparametric hypersurfaces turn out to be very complicated [Cal-4]. In the last ten years many people carried forward this research, see [Ab, FKM, Mü, OT], but the complete classification is still not known. Recently, the general theory of higher codimensional isoparametric submanifolds of Euclidean space and Hilbert space has been studied in [Ha, CW1-3, Te1-4, HPT, PT2].

Let $R^{n+k, 1}$ be the Lorentz space with the nondegenerate symmetric bilinear form $\langle x, y\rangle=\sum_{i=1}^{n+k} x_{i} y_{i}-x_{n+k+1} y_{n+k+1}$, and $H^{n+k}=\left\{x \in R^{n+k, 1} \mid\langle x, x\rangle=\right.$ $\left.-1, x_{n+k+1}>0\right\}$, the standard isometric embedding of hyperbolic space with sectional curvature -1 into $R^{n+k, 1}$. It is well known that any totally umbilic complete submanifold of $H^{n+k}$ is $L(V, u)=H^{n+k} \cap(V+u)$, where $V$ is a linear subspace of $R^{n+k, 1}$ and $u \in R^{n+k, 1}$. In fact, if $V \subset R^{n+k, 1}$ is a Euclidean subspace, i.e., $\langle\rangle \mid$,$V is positive definite, then L(V, u)$ is a sphere with sectional curvature $-1 /(\langle u, u\rangle+1)$, where $u \perp V$; if $V \subset R^{n+k, 1}$ is a Lorentz subspace, i.e., $\langle\rangle \mid$,$V is a nondegenerate symmetric bilinear form with index 1$, then $L(V, u)$ is a hyperbolic space with sectional curvature $-1 /(\langle u, u\rangle+1)$, where $u \perp V$; if $V \subset R^{n+k, 1}$ is degenerate, i.e., $\langle\rangle$,$V is a degenerate$ symmetric bilinear form, then $L(V, u)$ is flat and isometric to a Euclidean space. We will call $L(V, u)$ spherical if $V \subset R^{n+k, 1}$ is a Euclidean subspace, hyperbolic if $V \subset R^{n+k, 1}$ is a Lorentz subspace, and flat if $V \subset R^{n+k, 1}$ is degenerate.

Received by the editors February 9, 1990.

1980 Mathematics Subject Classification (1985 Revision). Primary 53C40; Secondary 57S25. 
A submanifold $M^{n}$ of $H^{n+k}$ is called isoparametric if it has a globally flat normal bundle and the principal curvatures along any parallel normal vector field are constant. In this paper we study isoparametric submanifolds of hyperbolic space and show that any isoparametric submanifold $M^{n}$ of $H^{n+k}$ is either an isoparametric submanifold of a totally umbilic hypersurface of $H^{n+k}$, or a standard product of an isoparametric submanifold of a spherical umbilic submanifold and a hyperbolic umbilic submanifold in $H^{n+k}$. As a consequence we are able to classify all polar actions of connected, closed Lie subgroups of $O(n, 1)$ on $H^{n}$.

Let $V$ be a separable Hilbert space. Let $\widetilde{V}=R \oplus V$ with the inner product

$$
\langle(s, x),(t, y)\rangle=-s t+\langle x, y\rangle \quad \text { where } s, t \in R, x, y \in V .
$$

Then $\widetilde{V}$ is a Lorentz Hilbert space. Let $H(\widetilde{V})=\left\{(s, x) \in \widetilde{V} \mid-s^{2}+\langle x, x\rangle=\right.$ $-1, s>0\}$. It is well known that $H(\widetilde{V})$ is a Riemannian Hilbert hyperbolic space with constant sectional curvature -1 . In this paper we prove that if $M$ is a submanifold of a Hilbert hyperbolic space $H(\widetilde{V})$ with finite codimension, and $M$ satisfies: (1) $\nu(M)$, the normal bundle of $M$ in $H(\widetilde{V})$, is globally flat, (2) for any parallel normal vector field $v$, the shape operator $A_{v(x)}$ is orthogonally equivalent to the shape operator $A_{v(y)}$ for all $x, y \in M,(3)$ the shape operator $A_{v}$ is a compact operator for any $v \in \nu(M)_{x}$, then $M$ is either of finite dimension, or a totally geodesic submanifold of $H(\widetilde{V})$, i.e., $M=H(\widetilde{V}) \cap V_{1}$, where $V_{1}$ is a Lorentz Hilbert subspace of $\widetilde{V}$.

This paper is organized as follows: in $\S 1$ we prove the basic properties of isoparametric submanifolds of hyperbolic space, and the decomposition theorem is given in $\S 2$. In $\S 3$ we obtain all polar actions of connected closed Lie subgroups of $O(n, 1)$ on $H^{n}$, and in $\S 4$, we study the infinite dimensional case.

The author would like to thank Professor R. S. Palais and Professor C. L. Terng for their encouragement and many valuable suggestions.

\section{BASIC PROPERTIES}

Suppose a submanifold $M^{n}$ of $H^{n+k}$ is isoparametric, i.e., the normal bundle of $M$ in $H^{n+k}$ is globally flat and the principal curvatures along any parallel normal vector field are constant. Let $\nu(M)$ be the normal bundle of $M$ in $R^{n+k, 1}$. Then $\nu(M)$ is globally flat, hence $\left\{A_{v} \mid v \in \nu(M)_{q}\right\}$ is a family of commuting selfadjoint operators on $T M_{q}$ for any $q \in M$. So, there exists a common eigendecomposition $T M_{q}=\bigoplus_{i=1}^{p} E_{i}(q)$, at $q$. Since the principal curvatures along any parallel normal vector field are constant, the $E_{i}$, $i=1,2, \ldots, p$ are smooth distributions and there exist $v_{1}, v_{2}, \ldots, v_{p}$, parallel normal vector fields, such that for any normal vector $v \in \nu(M)_{q}$, we have

$$
A_{v} \mid E_{i}=\left\langle v, v_{i}\right\rangle \mathrm{id}_{E_{i}} .
$$

The $E_{i}$ are called the curvature distributions of $M$, and the $v_{i}$ are called the curvature normals of $M$ in $R^{n+k, 1}$.

1.1. We will make the following standing assumptions:

(1) $M$ has $p$ curvature distributions, $E_{1}, E_{2}, \ldots, E_{p}$, and $\operatorname{rank}\left(E_{i}\right)=$ $m_{i}$. 
(2) Let $\left\{e_{j} \mid 1 \leq j \leq n\right\}$ be a local orthonormal tangent frame for $M$, such that $E_{i}$ is spanned by $\left\{e_{j} \mid \mu_{i-1}<j \leq \mu_{i}\right\}$, where $\mu_{i}=\sum_{s=1}^{i} m_{s}$, $\mu_{0}=0$.

(3) Let $\left\{e_{\alpha} \mid n+1 \leq \alpha \leq n+k+1\right\}$ be a local orthonormal parallel normal frame for $M$ in $R^{n+k, 1}$, where $e_{n+k+1}(x)=x$ for $x \in M$.

Remark. (a) Since $M^{n} \subset H^{n+k}$, we have $A_{q}=-I$ on $T_{q} M$. Hence there is a smooth vector field $u_{i}$ on $M$, such that $v_{i}(q)=u_{i}(q)+q$.

(b) Since the orthonormal frame field $\left\{e_{\alpha}\right\}$ for $M$ is parallel, we have

$$
\begin{gathered}
\omega_{\alpha \beta}=0 \text { for all } \alpha, \beta, \text { and } \\
\omega_{\alpha j}=-\left\langle v_{i}, e_{\alpha}\right\rangle \omega_{j} \quad \text { where } \mu_{i-1}<j \leq \mu_{i} .
\end{gathered}
$$

1.2 Definition. A submanifold $M$ of $H^{n+k}$ is called full if $M$ is not included in any totally umbilic hypersurface of $H^{n+k}$.

1.3 Proposition. Let $L(V, u) \subset H^{n+k}$ be a totally umbilic hypersurface and suppose $M \subset L(V, u) \subset H^{n+k}$. Then $M$ is isoparametric in $L(V, u)$ iff $M$ is isoparametric in $H^{n+k}$.

Proof. If $M$ is isoparametric in $H^{n+k}$, then it is obvious that $M$ is isoparametric in $L(V, u)$.

Suppose $M$ is isoparametric in $L(V, u)$. If $V=\left\{x \in R^{n+k, 1} \mid\langle x, v\rangle=0\right\}$, then $L(V, u)=\left\{x \in H^{n+k} \mid\langle x, v\rangle=a\right\}$, where $a=\langle u, v\rangle$. Suppose $e_{n+k}$ is a normal vector field of $L(V, u)$ in $H^{n+k}$, and $X: H^{n+k} \rightarrow R^{n+k, 1}$ is the standard isometric embedding. Then $v=-a X+b e_{n+k}$, so $0=-a \mathrm{~d} X+$ $b \mathrm{~d} e_{n+k}$. For any tangent vector field $Z$ on $M$, we have, $b \mathrm{~d} e_{n+k}(Z)=a Z$. Since $b \neq 0, e_{n+k}$ is a parallel normal vector field and $A_{e_{n+k}}$ has constant eigenvalues on $M$. Therefore the normal bundle of $M$ in $H^{n+k}$ is flat and the principal curvatures along any parallel normal vector field are constant, i.e., $M$ is isoparametric in $H^{n+k}$.

1.4 Proposition. $M$ is full in $H^{n+k}$ iff $v_{1}, v_{2}, \ldots, v_{p}$ span $\nu(M)$.

Proof. If $M$ is not full in $H^{n+k}$ then $M$ is included in a totally umbilic hypersurface of $H^{n+k}$, i.e., there exists $v \in R^{n+k, 1}, v \neq 0$, such that $\langle v, x\rangle$ is constant on $M$. Hence $v$ is a constant normal field on $M$, we have $A_{v}=0$. On the other hand we know $A_{v} \mid E_{i}=\left\langle v, v_{i}\right\rangle \operatorname{id}_{E_{i}}$. Therefore $\left\langle v, v_{i}\right\rangle=0$, $i=1,2, \ldots, p$. Since $v \neq 0, v_{1}, v_{2}, \ldots, v_{p}$ do not span $\nu(M)$.

If $v_{1}, v_{2}, \ldots, v_{p}$ do not span $\nu(M)$, then there exists a normal field $v$ on $M$, such that $\left\langle v, v_{i}\right\rangle=0, i=1,2, \ldots, p$, and we have $A_{v} \mid E_{i}=\left\langle v, v_{i}\right\rangle \operatorname{id}_{E_{i}}=$ $0, i=1,2, \ldots, p, A_{v}=0$; i.e., $v$ is constant on $M$. Then

$$
\mathrm{d}\langle v, x\rangle=\langle v, \mathrm{~d} x\rangle=\sum_{i=1}^{n} \omega_{i}\left\langle v, e_{i}\right\rangle=0,
$$

hence $\langle v, x\rangle=c$ is constant, i.e., $M$ is included in a totally umbilic hypersurface of $H^{n+k}$.

1.5 Lemma. If $\omega_{i j}=\sum_{k} \gamma_{i j}^{k} \omega_{k}$ then

$$
\gamma_{i j}^{k}\left(v_{i^{\prime}}-v_{j^{\prime}}\right)=\gamma_{i k}^{j}\left(v_{i^{\prime}}-v_{k^{\prime}}\right)=\gamma_{k j}^{i}\left(v_{k^{\prime}}-v_{j^{\prime}}\right)
$$

where $\mu_{i^{\prime}-1}<i \leq \mu_{i^{\prime}}, \mu_{j^{\prime}-1}<j \leq \mu_{j^{\prime}}, \mu_{k^{\prime}-1}<k \leq \mu_{k^{\prime}}$, and $j \neq k$. 
Proof. Suppose $\mu_{i^{\prime}-1}<i \leq \mu_{i^{\prime}}$. Then we have $\omega_{\alpha i}=-\left\langle v_{i^{\prime}}, e_{\alpha}\right\rangle \omega_{i}$ for all $\alpha$, and because $\mathrm{d} \omega_{\alpha i}=\omega_{\alpha j} \wedge \omega_{j i}$, we have

$$
\begin{aligned}
& \omega_{\alpha j} \wedge \omega_{j i}=-\left\langle v_{i^{\prime}}, e_{\alpha}\right\rangle \mathrm{d} \omega_{i}=-\left\langle v_{i^{\prime}}, e_{\alpha}\right\rangle \omega_{i j} \wedge \omega_{j}, \\
& \omega_{i j} \wedge\left\langle v_{j^{\prime}}, e_{\alpha}\right\rangle \omega_{j}=\left\langle v_{i^{\prime}}, e_{\alpha}\right\rangle \omega_{i j} \wedge \omega_{j}, \\
& \sum_{k} \gamma_{i j}^{k}\left\langle v_{j^{\prime}}, e_{\alpha}\right\rangle \omega_{k} \wedge \omega_{j}=\sum_{k} \gamma_{i j}^{k}\left\langle v_{i^{\prime}}, e_{\alpha}\right\rangle \omega_{k} \wedge \omega_{j} .
\end{aligned}
$$

Comparing coefficients of $\omega_{k} \wedge \omega_{j}$ for $j \neq k$, if $\omega_{k^{\prime}-1}<k \leq \omega_{k^{\prime}}$ we have

$$
\begin{gathered}
\gamma_{i j}^{k}\left\langle v_{j^{\prime}}, e_{\alpha}\right\rangle-\gamma_{i k}^{j}\left\langle v_{k^{\prime}}, e_{\alpha}\right\rangle=\gamma_{i j}^{k}\left\langle v_{i^{\prime}}, e_{\alpha}\right\rangle-\gamma_{i k}^{j}\left\langle v_{i^{\prime}}, e_{\alpha}\right\rangle \quad \forall \alpha, \\
\gamma_{i j}^{k}\left\langle v_{i^{\prime}}-v_{j^{\prime}}, e_{\alpha}\right\rangle=\gamma_{i k}^{j}\left\langle v_{i^{\prime}}-v_{k^{\prime}}, e_{\alpha}\right\rangle \quad \forall \alpha,
\end{gathered}
$$

hence, $\gamma_{i j}^{k}\left(v_{i^{\prime}}-v_{j^{\prime}}\right)=\gamma_{i k}^{j}\left(v_{i^{\prime}}-v_{k^{\prime}}\right)=\gamma_{k j}^{i}\left(v_{k^{\prime}}-v_{j^{\prime}}\right)$.

1.6 Corollary. Suppose $\mu_{i^{\prime}-1}<i \leq \mu_{i^{\prime}}, \mu_{j^{\prime}-1}<j \leq \mu_{j^{\prime}}$, and $i^{\prime} \neq j^{\prime}$. Then

(1) $\gamma_{i j}^{k}=0$, if $\mu_{j^{\prime}-1}<k \leq \mu_{j^{\prime}}$.

(2) If $\gamma_{i j}^{k} \neq 0$, where $\mu_{k^{\prime}-1}<k \leq \mu_{k^{\prime}}$, then

$$
\begin{aligned}
& v_{k^{\prime}}=\left(1-a_{i^{\prime} j^{\prime}}^{k^{\prime}}\right) v_{i^{\prime}}+\alpha_{i^{\prime} j^{\prime}}^{k^{\prime}} v_{j^{\prime}}, \quad \text { where } a_{i^{\prime} j^{\prime}}^{k^{\prime}} \neq 0,1, \\
& \gamma_{i j}^{k}=\gamma_{i k}^{j} a_{i^{\prime} j^{\prime}}^{k^{\prime}}=\gamma_{k j}^{i}\left(1-a_{i^{\prime} j^{\prime}}^{k^{\prime}}\right) .
\end{aligned}
$$

1.7 Proposition. Each curvature distribution $E_{i}$ is integrable.

Proof. For simplicity, we consider the case $i=1$. Since $E_{1}$ is defined by the 1-form equations on $M: \omega_{i}=0, m_{1}<i \leq n$, and

$$
\begin{aligned}
\mathrm{d} \omega_{i} & =\sum_{j=1}^{n} \omega_{i j} \wedge \omega_{j}=\sum_{j \leq m_{1}} \omega_{i j} \wedge \omega_{j} \\
& =\sum_{\substack{j \leq m_{1} \\
k \leq m_{1}}} \gamma_{i j}^{k} \omega_{k} \wedge \omega_{j}=0 \text { by (1.6). }
\end{aligned}
$$

It follows that $E_{1}$ is integrable.

1.8. Recall that the endpoint map $Y: \nu(M) \rightarrow R^{n+k, 1}$ is defined by $Y(v)=$ $x+v$ for $v \in \nu(M)_{x}$, and that a singular value of $Y$ is called a focal point of $M$.

1.9 Proposition. Let $M$ be an isoparametric submanifold of $H^{n+k} \subset R^{n+k, 1}$ and $\Gamma$ its focal point set. For each $q \in M$, let $\Gamma_{q}$ denote the intersection of $\Gamma$ with the normal plane $\nu(M)_{q}$ to $M$ at $q$. Then

$$
\Gamma=\bigcup_{q \in M} \Gamma_{q} \text { and } \Gamma_{q}=\bigcup_{i=1}^{p} l_{i}(q),
$$

where $l_{i}(q)=\left\{v \in \nu(M)_{q} \mid\left\langle v, v_{i}\right\rangle=0\right\}$. These $l_{i}(q)$ are called the focal hyperplanes associated to $E_{i}$ at $q$.

Proof. Let $Y$ be the endpoint map,

$$
Y: \nu(M) \rightarrow R^{n+k, 1}, \quad(x, v) \mapsto x+v,
$$


so, we have

$$
\begin{aligned}
Y & =Y(x, z)=x+\sum_{\alpha} z_{\alpha} e_{\alpha} \\
\mathrm{d} Y & =\mathrm{d} x+\sum_{\alpha} \mathrm{d} z_{\alpha} e_{\alpha}+\sum_{\alpha} z_{\alpha} \mathrm{d} e_{\alpha} \\
& =I-A \sum_{\alpha} z_{\alpha} e_{\alpha}+\sum_{\alpha} \mathrm{d} z_{\alpha} e_{\alpha} \\
& =\bigoplus_{i=1}^{p}\left(1-\left\langle\sum_{\alpha} z_{\alpha} e_{\alpha}, v_{i}\right\rangle\right) \mathrm{id}_{E_{i}}+\sum_{\alpha} \mathrm{d} z_{\alpha} e_{\alpha} .
\end{aligned}
$$

Then

$$
\Gamma=\bigcup_{q \in M} \Gamma_{q}
$$

and

$$
\begin{aligned}
\Gamma_{q} & =\bigcup_{i=1}^{p}\left\{q+v \mid v \in \nu(M)_{q},\left\langle v, v_{i}\right\rangle=1\right\} \\
& =\bigcup_{i=1}^{p}\left\{v \mid v \in \nu(M)_{q},\left\langle v, v_{i}\right\rangle=0\right\} \\
& =\bigcup_{i=1}^{p} l_{i}(q),
\end{aligned}
$$

where $l_{i}(q)=\left\{v \in \nu(M)_{q} \mid\left\langle v, v_{i}\right\rangle=0\right\}$.

1.10 Proposition. Let $X: M^{n} \subset H^{n+k} \subset R^{n+k, 1}$ be an isoparametric submanifold, and $v$ a parallel normal vector field on $M$.

Then $X+v: M \rightarrow R^{n+k, 1}$ is an immersion if and only if $\left\langle v, v_{i}\right\rangle \neq 1$ for $i=1,2, \ldots, p$. Moreover, if $X+v$ is an immersion, then

(i) The parallel set $M_{v}=\{x+v \mid x \in M\}$ is an isoparametric submanifold of $R^{n+k, 1}$, i.e., $M_{v}$ is a space-like submanifold of $R^{n+k, 1}$, the normal bundle of $M_{v}$ in $R^{n+k, 1}$ is globally flat, and the principal curvatures along any parallel normal field are constant.

(ii) Let $q^{*}=q+v(q)$, then

$$
\begin{gathered}
T M_{q}=T\left(M_{v}\right)_{q^{*}}, \quad \nu\left(M_{q}\right)=\nu\left(M_{v}\right)_{q^{*}}, \\
q+\nu(M)_{q}=q^{*}+\nu\left(M_{v}\right)_{q^{*}} .
\end{gathered}
$$

(iii) If $\left\{e_{\alpha}\right\}$ is a local parallel normal frame on $M$, then $\left\{\bar{e}_{\alpha}\right\}$ is a local parallel normal frame on $M_{v}$, where $\bar{e}_{\alpha}\left(q^{*}\right)=e_{\alpha}(q)$.

(iv) $E_{i}^{*}\left(q^{*}\right)=E_{i}(q)$ are the curvature distributions of $M_{v}$, and the corresponding curvature normals are given by

$$
v_{i}^{*}\left(q^{*}\right)=\frac{v_{i}(q)}{1-\left\langle v, v_{i}\right\rangle} .
$$

(v) The focal hyperplane $l_{i}^{*}\left(q^{*}\right)$ of $M_{v}$ associated to $E_{i}^{*}$ is the same as the focal hyperplane $l_{i}(q)$ of $M$ associated to $E_{i}$. 
Proof. The same proof as for Euclidean space works here. For details see [PT2, Te1].

1.11 Theorem. Let $M$ be a complete isoparametric submanifold of $H^{n+k} \subset$ $R^{n+k, 1}, E_{i}$ the curvature distributions, $v_{i}$ the curvature normals, and $l_{i}(q)$ the focal hyperplanes associated to $E_{i}$ at $q \in M$. Let $S_{i}(q)$ denote the leaf of $E_{i}$ through $q$.

(1) If $\left\langle v_{i}, v_{i}\right\rangle>0$, then

(i) $E_{i}(x) \oplus \nu(M)_{x}$ is a fixed $m_{i}+k+1$ Lorentz subspace $\eta_{i}$ in $R^{n+k, 1}$ for all $x \in S_{i}(q)$.

(ii) $E_{i}(x) \oplus R v_{i}(x)$ is a fixed $m_{i}+1$ Euclidean subspace $\xi_{i}$ in $R^{n+k, 1}$ for all $x \in S_{i}(q)$.

(iii) $l_{i}(x)=l_{i}(q)$ for all $x \in S_{i}(q)$.

(iv) $x+v_{i}(x) /\left\langle v_{i}, v_{i}\right\rangle=c_{i}$ is a constant for all $x \in S_{i}(q)$.

(v) $S_{i}(q)$ is the standard sphere of $\xi_{i}+c_{i}$, with radius $1 /\left|v_{i}\right|$ and centered at $c_{i}$.

(2) If $\left\langle v_{i}, v_{i}\right\rangle<0$, then

(i) $E_{i}(x) \oplus \nu(M)_{x}$ is a fixed $m_{i}+k+1$ Lorentz subspace $\eta_{i}$ in $R^{n+k, 1}$ for all $x \in S_{i}(q)$.

(ii) $E_{i}(x) \oplus R v_{i}(x)$ is a fixed $m_{i}+1$ Lorentz subspace $\xi_{i}$ in $R^{n+k, 1}$ for all $x \in S_{i}(q)$.

(iii) $l_{i}(x)=l_{i}(q)$ for all $x \in S_{i}(q)$.

(iv) $x+v_{i}(x) /\left\langle v_{i}, v_{i}\right\rangle=c_{i}$ is a constant for all $x \in S_{i}(q)$.

(v) $S_{i}(q)$ is the standard hyperbolic space of $\xi_{i}+c_{i}$, with radius $1 /\left|v_{i}\right|$ and centered at $c_{i}$, i.e.,

$$
S_{i}(q)=\left\{x \in c_{i}+\xi_{i} \mid\left\langle x-c_{i}, x-c_{i}\right\rangle=1 /\left\langle v_{i}, v_{i}\right\rangle\right\}
$$

(3) If $\left\langle v_{i}, v_{i}\right\rangle=0$, then

(i) $v_{i}(x)=c_{i}$ is a constant on $S_{i}(q)$.

(ii) $E_{i}(x) \oplus R v_{i}(x)+R x$ is a fixed $m_{i}+2$ Lorentz subspace $\zeta_{i}$ for $x \in S_{i}(q)$.

(iii) $S_{i}(q)$ is a flat umbilic hypersurface of the hyperbolic space $H^{n+k} \cap$ $\zeta_{i}$.

Proof. The proof for (1) and (2) is the same as for Euclidean space. For details see [PT2, Te1].

Proof of (3). For simplicity, we assume $i=1$.

(i) Since $\nabla_{e_{j}} v_{1}=A_{v_{1}} e_{j}=\left\langle v_{1}, v_{1}\right\rangle=0$, if $e_{j} \in E_{1}$, so $v_{1}(x)=c_{1}$ is constant on $S_{1}(q)$.

(ii) Let $v_{1}(x)=u_{1}(x)+x$. Then, we have

$$
E_{1}(x) \oplus R v_{1}+R x=E_{1}(x) \oplus R u_{1} \oplus R x,
$$




$$
\begin{aligned}
& \mathrm{d}\left(e_{1} \wedge \cdots \wedge e_{m_{1}} \wedge u_{1} \wedge x\right)=\sum_{j} e_{1} \wedge \cdots \wedge \mathrm{d} e_{j} \wedge \cdots \wedge e_{m_{1}} \wedge u_{1} \wedge x \\
& =\sum_{j} e_{1} \wedge \cdots \wedge\left(\sum_{k} \omega_{j k} e_{k}+\sum_{\alpha} \omega_{j \alpha} e_{\alpha}\right) \wedge \cdots \wedge e_{m_{1}} \wedge u_{1} \wedge x \\
& =\sum_{j \alpha} e_{1} \wedge \cdots \wedge \omega_{j \alpha} e_{\alpha} \wedge \cdots \wedge u_{1} \wedge x \\
& =\sum_{j} e_{1} \wedge \cdots \wedge \sum_{\alpha \leq n+k} \omega_{j \alpha} e_{\alpha} \wedge \cdots \wedge u_{1} \wedge x \\
& \quad=\sum_{j} \omega_{j} e_{1} \wedge \cdots \wedge \sum_{\alpha \leq n+k}\left\langle v_{1}, e_{\alpha}\right\rangle e_{\alpha} \wedge \cdots \wedge u_{1} \wedge x \\
& =0 .
\end{aligned}
$$

So $\zeta_{1}=E_{1}(x) \oplus R v_{1}+R x$ is a fixed $m_{1}+2$ Lorentz subspace for all $x \in$ $S_{1}(q)$.

(iii) For any $x \in S_{1}(q)$, we have

$$
\langle x, x\rangle=-1, \quad\left\langle x, c_{1}\right\rangle=-1 \text { and } x \in \zeta_{1},
$$

so $S_{1}(q)$ is a flat umbilic hypersurface in the hyperbolic space of $H^{n+k} \cap$ $\zeta_{1}$.

1.12 Definition. If $v \in R^{n+k, 1},\langle v, v\rangle \neq 0$, then $R_{v}(x)=x-2\langle x, v\rangle v /\langle v, v\rangle$ $\in O(n+k, 1)$ is called the linear reflection along $v$.

1.13. Suppose $M^{n} \subset H^{n+k}$ is an isoparametric submanifold with curvature normals $v_{1}, v_{2}, \ldots, v_{p}$, where $\left\langle v_{i}, v_{i}\right\rangle \leq 0$ if $i=1,2, \ldots, l$ and where $\left\langle v_{i}, v_{i}\right\rangle>0$ if $i=l+1, \ldots, p$.

Some notations:

(1) $R_{i}^{q}$ denotes the linear reflection of $\nu(M)_{q}$ along $v_{i}(q), i=l+1, \ldots, p$.

(2) Let $\varphi_{i}$ be the diffeomorphism of $M$ defined by $\varphi_{i}(q)=$ the antipodal point of $q$ in the leaf sphere $S_{i}(q)$ of $E_{i}$ for $i=l+1, \ldots, p$.

(3) $S_{p}$ denotes the group of permutations of $\{1,2, \ldots, p\}$.

It follows from (1) of (1.11) that

$$
\varphi_{i}=X+2 \frac{v_{i}}{\left\langle v_{i}, v_{i}\right\rangle}, \quad i=l+1, \ldots, p
$$

and $\varphi_{i}(q)=R_{i}^{q}(q)$. Since $\varphi_{i}$ is a diffeomorphism, it follows from (1.10) that

1.14 Proposition. If $i \geq l+1$, then $2\left(v_{i} /\left\langle v_{i}, v_{i}\right\rangle, v_{j}\right\rangle \neq 1$ for all $j$, i.e.,

$$
1-2\left\langle v_{i}, v_{j}\right\rangle /\left\langle v_{i}, v_{i}\right\rangle \neq 0 \text { for all } j \text {. }
$$

1.15 Theorem. There exist permutations $\sigma_{l+1}, \ldots, \sigma_{p} \in S_{p}$ such that

$$
E_{j}\left(\varphi_{i}(q)\right)=E_{\sigma_{i}(j)}(q), \text { i.e., } \varphi_{i}^{*}\left(E_{j}\right)=E_{\sigma_{i}(j)} .
$$

In particular, $m_{j}=m_{\sigma_{i}(j)}$.

$$
\begin{gathered}
v_{\sigma_{i}(j)}(q)=\left(1-2 \frac{\left\langle v_{i}, v_{\sigma_{i}(j)}\right\rangle}{\left\langle v_{i}, v_{i}\right\rangle}\right) v_{j}\left(\varphi_{i}(q)\right) \text { and } \\
R_{i}^{q}\left(v_{j}(q)\right)=\left(1-2 \frac{\left\langle v_{i}, v_{\sigma_{i}(j)}\right\rangle}{\left\langle v_{i}, v_{i}\right\rangle}\right)^{-1} v_{\sigma_{i}(j)}(q),
\end{gathered}
$$

where $i \geq l+1,1 \leq j \leq p$. 
Proof. The same proof as for Euclidean space with trivial modifications.

1.16 Corollary. The subgroup $W^{q}$ of $O(\nu(M))_{q}$ generated by $R_{v_{i}}^{q}, \quad i=l+$ $1, \ldots, p$, is a finite group.

\section{The Decomposition Theorem}

Suppose $M^{n} \subset H^{n+k} \subset R^{n+k, 1}$ is a full isoparametric submanifold with curvature normals $v_{1}, v_{2}, \ldots, v_{p}$ such that

$\left\langle v_{i}, v_{i}\right\rangle \leq 0$ for $i=1, \ldots, l$ and $\left\langle v_{i}, v_{i}\right\rangle>0$ for $i=l+1, \ldots, p$.

2.1 Lemma. Suppose $\mu_{i^{\prime}-1}<i \leq \mu_{i^{\prime}}, \mu_{j^{\prime}-1}<j \leq \mu_{j^{\prime}}$, and $i^{\prime} \neq j^{\prime}$ then

$$
\left\langle v_{i^{\prime}}, v_{j^{\prime}}\right\rangle=\sum_{\substack{k^{\prime} \\ v_{k^{\prime}}=\left(1-a_{i^{\prime} j^{\prime}}^{k^{\prime}}\right) v_{i^{\prime}+a_{i^{\prime} j^{\prime}}^{k^{\prime}} v_{j^{\prime}}}^{k_{i^{\prime} j^{\prime}} \neq 0,1}}} \sum_{\mu_{k^{\prime}-1}<k \leq \mu_{k^{\prime}}}\left(\gamma_{i j}^{k}\right)^{2} \frac{1}{a_{i^{\prime} j^{\prime}}^{k^{\prime}}\left(a_{i^{\prime} j^{\prime}}^{k^{\prime}}-1\right)} .
$$

Proof. By the Gauss equation

$$
-\mathrm{d} \omega_{i j}+\omega_{i k} \wedge \omega_{k j}+\sum_{\alpha \leq n+k} \omega_{i \alpha} \wedge \omega_{\alpha j}=-\omega_{i} \wedge \omega_{j}
$$

We have

$$
\begin{gathered}
-\sum_{k} \mathrm{~d}\left(\gamma_{i j}^{k} \omega_{k}\right)+\sum_{k t m} \gamma_{i t}^{k} \omega_{k} \wedge \gamma_{t j}^{m} \omega_{m} \\
-\sum_{\alpha \leq n+k}\left\langle v_{i^{\prime}}, e_{\alpha}\right\rangle\left\langle v_{j^{\prime}}, e_{\alpha}\right\rangle \omega_{i} \wedge \omega_{j}=-\omega_{i} \wedge \omega_{j} \\
-\sum_{k} \mathrm{~d} \gamma_{i j}^{k} \wedge \omega_{k}-\sum_{k t} \gamma_{i j}^{k} \omega_{k t} \wedge \omega_{t}+\sum_{k t m} \gamma_{i t}^{k} \gamma_{t j}^{m} \omega_{k} \wedge \omega_{m} \\
=\left(\sum_{\alpha \leq n+k}\left\langle v_{i^{\prime}}, e_{\alpha}\right\rangle\left\langle v, e_{\alpha}\right\rangle-1\right) \omega_{i} \wedge \omega_{j}=\left\langle v_{i^{\prime}}, v_{j^{\prime}}\right\rangle \omega_{i} \wedge \omega_{j}
\end{gathered}
$$

Comparing the coefficients of $\omega_{i} \wedge \omega_{j}$, we have,

$$
\begin{gathered}
-\sum_{k} \gamma_{i j}^{k} \gamma_{k j}^{i}+\sum_{k} \gamma_{i j}^{k} \gamma_{k i}^{j}+\sum_{k} \gamma_{i k}^{i} \gamma_{k j}^{j}-\sum_{k} \gamma_{i k}^{j} \gamma_{k j}^{i}=\left\langle v_{i^{\prime}}, v_{j^{\prime}}\right\rangle, \\
\sum_{\substack{k \\
e_{k} \notin E_{i^{\prime}}, E_{j^{\prime}}}}\left(\gamma_{i j}^{k} \gamma_{k i}^{j}-\gamma_{i j}^{k} \gamma_{k j}^{i}-\gamma_{i k}^{j} \gamma_{k j}^{i}\right)=\left\langle v_{i^{\prime}}, v_{j^{\prime}}\right\rangle \quad \text { (by (1.6)). }
\end{gathered}
$$

By (2) of (1.6), we have

$$
\begin{aligned}
& \sum_{\substack{k^{\prime} \\
v_{k^{\prime}}=\left(1-a_{i^{\prime} j^{\prime}}^{k^{\prime}}\right) \\
a_{i^{\prime} j^{\prime}}^{k^{\prime}} \neq 0,1}} \sum_{\substack{\mu_{k^{\prime}-1}<k \leq a_{i^{\prime} j^{\prime}}^{k^{\prime}}, v_{j^{\prime}} \\
=}}\left\{-\frac{\left(\gamma_{i j}^{k}\right)^{2}}{a_{i^{\prime} j^{\prime}}^{k^{\prime}}}-\frac{\left(\gamma_{i j}^{k}\right)^{2}}{1-a_{i^{\prime} j^{\prime}}^{k^{\prime}}}-\frac{\left(\gamma_{i j}^{k}\right)^{2}}{a_{i^{\prime} j^{\prime}}^{k^{\prime}}\left(1-a_{i^{\prime} j^{\prime}}^{k^{\prime}}\right)}\right\} \\
& =\left\langle v_{i^{\prime}}, v_{j^{\prime}}\right\rangle,
\end{aligned}
$$




$$
\sum_{\substack{v_{k^{\prime}}=\left(1-a_{i^{\prime} j^{\prime}}^{k^{\prime}}\right) v_{i^{\prime}}+a_{i^{\prime} j^{\prime}}^{k^{\prime}} v_{j^{\prime}} \\ a_{i^{\prime} j^{\prime}} \neq 0,1}} \sum_{\mu_{k^{\prime}-1}<k \leq \mu_{k^{\prime}}}\left(\gamma_{i j}^{k}\right)^{2} \frac{1}{a_{i^{\prime} j^{\prime}}^{k^{\prime}}\left(a_{i^{\prime} j^{\prime}}^{k^{\prime}}-1\right)}=\left\langle v_{i^{\prime}}, v_{j^{\prime}}\right\rangle .
$$

\subsection{Lemma. Suppose $1 \leq i, j \leq l$. Then,}

$$
\left\langle v_{i}, v_{j}\right\rangle<0 \text { if } i \neq j,
$$

(2) $\left\langle v_{i}, v_{j}\right\rangle^{2} \geq\left\langle v_{i}, v_{i}\right\rangle\left\langle v_{j}, v_{j}\right\rangle$ and $\left\langle v_{i}, v_{j}\right\rangle^{2}=\left\langle v_{i}, v_{i}\right\rangle\left\langle v_{j}, v_{j}\right\rangle \quad$ iff $i=j$.

Proof. (1) Suppose $q \in M$, then $v_{i}(q)=u_{i}+q$, where $\left\langle q, u_{i}\right\rangle=0, i=$ $1, \ldots, l$. So we have $\left\langle v_{i}, v_{j}\right\rangle=\left\langle u_{i}, u_{j}\right\rangle-1, \forall i, j$. Since $\left\langle v_{i}, v_{i}\right\rangle \leq 0$, $\left\langle u_{i}, u_{i}\right\rangle \leq 1$, and (1) follows from the Schwarz inequality.

(2) If $\left\langle v_{i}, v_{i}\right\rangle=0$, then by (1) we have $\left\langle v_{i}, v_{j}\right\rangle^{2}>0=\left\langle v_{i}, v_{i}\right\rangle\left\langle v_{j}, v_{j}\right\rangle$ if $i \neq j$. If $\left\langle v_{i}, v_{i}\right\rangle<0$, then, since

$$
\left\langle v_{j}-\frac{\left\langle v_{i}, v_{j}\right\rangle}{\left\langle v_{i}, v_{i}\right\rangle} v_{i}, v_{i}\right\rangle=0
$$

it follows that

$$
\left\langle v_{j}-\frac{\left\langle v_{i}, v_{j}\right\rangle}{\left\langle v_{i}, v_{i}\right\rangle} v_{i}, v_{j}-\frac{\left\langle v_{i}, v_{j}\right\rangle}{\left\langle v_{i}, v_{i}\right\rangle} v_{i}\right\rangle \geq 0
$$

hence

$$
\left\langle v_{j}, v_{j}\right\rangle-\frac{\left\langle v_{i}, v_{j}\right\rangle^{2}}{\left\langle v_{i}, v_{i}\right\rangle}=\left\langle v_{j}-\frac{\left\langle v_{i}, v_{j}\right\rangle}{\left\langle v_{i}, v_{i}\right\rangle} v_{i}, v_{j}-\frac{\left\langle v_{i}, v_{j}\right\rangle}{\left\langle v_{i}, v_{i}\right\rangle} v_{i}\right\rangle \geq 0
$$

Therefore we have

$$
\left\langle v_{i}, v_{j}\right\rangle^{2} \geq\left\langle v_{i}, v_{i}\right\rangle\left\langle v_{j}, v_{j}\right\rangle
$$

and

$$
\left\langle v_{i}, v_{j}\right\rangle^{2}=\left\langle v_{i}, v_{i}\right\rangle\left\langle v_{j}, v_{j}\right\rangle \quad \text { iff } v_{j}=\frac{\left\langle v_{i}, v_{j}\right\rangle}{\left\langle v_{i}, v_{i}\right\rangle} v_{i}
$$

Since $\left\langle v_{i}(q), q\right\rangle=\left\langle v_{j}(q), q\right\rangle=-1$,

$$
\left\langle v_{i}, v_{j}\right\rangle^{2}=\left\langle v_{i}, v_{i}\right\rangle\left\langle v_{j}, v_{j}\right\rangle \quad \text { iff } i=j .
$$

2.3 Theorem. Suppose $v_{1}, \ldots, v_{p}$ are the curvature normals of $M$, where

$$
\left\langle v_{i}, v_{i}\right\rangle \leq 0, \quad i=1, \ldots, l, \quad\left\langle v_{i}, v_{i}\right\rangle>0, \quad i=l+1, \ldots, p,
$$

then $l \leq 1$.

Proof. Suppose $l>1$. Then we can choose $v_{i^{\prime}}, v_{j^{\prime}} i^{\prime}, j^{\prime} \leq l$ and $i^{\prime} \neq j^{\prime}$ such that

$$
\begin{array}{ll}
\left\langle v_{i^{\prime}}, v_{i^{\prime}}\right\rangle \geq\left\langle v_{k^{\prime}}, v_{k^{\prime}}\right\rangle & \text { for all } k^{\prime} \leq l, \\
\left\langle v_{i^{\prime}}, v_{j^{\prime}}\right\rangle \geq\left\langle v_{i^{\prime}}, v_{k^{\prime}}\right\rangle & \text { for all } k^{\prime} \leq l, k^{\prime} \neq i^{\prime}
\end{array}
$$

Claim. If $k^{\prime} \leq l, v_{k^{\prime}}=\left(1-a_{i^{\prime} j^{\prime}}^{k^{\prime}}\right) v_{i^{\prime}}+a_{i^{\prime} j^{\prime}}^{k^{\prime}}$ then, either $a_{i^{\prime} j^{\prime}}^{k^{\prime}} \leq 0$, or, $a_{i^{\prime} j^{\prime}}^{k^{\prime}} \geq 1$. Otherwise, suppose $0<a_{i^{\prime} j^{\prime}}^{k^{\prime}}<1$. Then, we have

$$
\begin{aligned}
\left\langle v_{i^{\prime}}, v_{k^{\prime}}\right\rangle & =\left(1-a_{i^{\prime} j^{\prime}}^{k^{\prime}}\right)\left\langle v_{i^{\prime}}, v_{i^{\prime}}\right\rangle+a_{i^{\prime} j^{\prime}}^{k^{\prime}}\left\langle v_{i^{\prime}}, v_{j^{\prime}}\right\rangle \\
& >\left(1-a_{i^{\prime} j^{\prime}}^{k^{\prime}}\right)\left\langle v_{i^{\prime}}, v_{j^{\prime}}\right\rangle+a_{i^{\prime} j^{\prime}}^{k^{\prime}}\left\langle v_{i^{\prime}}, v_{j^{\prime}}\right\rangle \text { by }(2.2) \\
& =\left\langle v_{i^{\prime}}, v_{j^{\prime}}\right\rangle .
\end{aligned}
$$

Contradiction. 
If $k^{\prime}>l$ and $v_{k^{\prime}}=\left(1-a_{i^{\prime} j^{\prime}}^{k^{\prime}}\right) v_{i^{\prime}}+a_{i^{\prime} j^{\prime}}^{k^{\prime}}$, then either $a_{i^{\prime} j^{\prime}}^{k^{\prime}} \leq 0$, or $a_{i^{\prime} j^{\prime}}^{k^{\prime}} \geq 1$ because $\left\langle v_{k^{\prime}}, v_{k^{\prime}}\right\rangle>0$.

By (2.1) we have

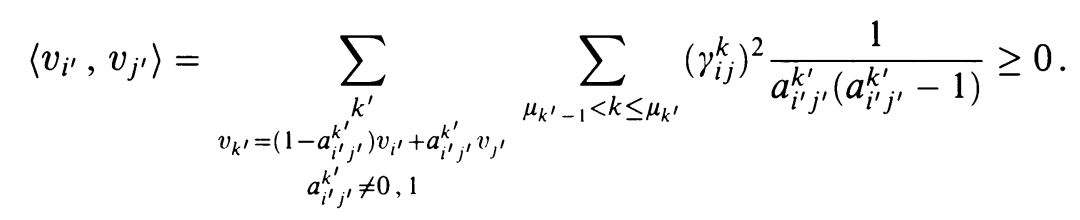

Contradiction. So, $l \leq 1$.

2.4 Theorem. Suppose $M^{n}$ is a full isoparametric submanifold of $H^{n+k} \subset$ $R^{n+k, 1}$ with curvature normals $v_{1}, v_{2}, \ldots, v_{p}$, where $\left\langle v_{i}, v_{i}\right\rangle \leq 0, i=1, \ldots$, $l$, and $\left\langle v_{i}, v_{i}\right\rangle>0, i=l+1, \ldots, p$.

Then $l=1$, and $\left\langle v_{1}, v_{i}\right\rangle=0, i>1$.

Proof. Since $M$ is full in $H^{n+k}, v_{1}(q), \ldots, v_{p}(q)$ span $\nu(M)_{q}$. Suppose there is no curvature normal with negative length. Then, by $(2.3)$, there are two cases:

(i) $\left\langle v_{1}, v_{1}\right\rangle=0$, and $\left\langle v_{i}, v_{i}\right\rangle>0, i>1$, or

(ii) $\left\langle v_{i}, v_{i}\right\rangle>0, i=1,2, \ldots, p$.

Case (i). Since $R_{v_{i}}^{q}\left(v_{1}\right)=c v_{\sigma_{i}(1)}$, where $c \in R$, and $R_{v_{i}}^{q} \in O\left(\nu(M)_{q}\right)$, we have $v_{\sigma_{i}(1)}=v_{1}$. Hence

$$
R_{v_{i}}^{q}\left(v_{1}\right)=c v_{1}, \quad v_{1}-2 \frac{\left\langle v_{i}, v_{1}\right\rangle}{\left\langle v_{i}, v_{i}\right\rangle}=c v_{1}
$$

This implies that $\left\langle v_{i}, v_{1}\right\rangle=0, i>1$, so, $v_{1}, \ldots, v_{p}$ do not span $\nu(M)_{q}$, a contradiction.

Case (ii). Let $u=\sum_{\tau \in W^{q}} \tau(q)$. Then $u \in \nu(M)_{q}$ is invariant under $W^{q}$. In particular $R_{v_{i}}^{q}(u)=u$ so $\left\langle v_{i}, u\right\rangle=0$.

Claim. $\tau(q), \forall \tau \in W^{q}$, are in the same component of the interior of the light cone, so $\langle u, u\rangle<0$.

Proof of the claim. Since $W^{q}$ is generated by $R_{v_{i}}^{q}$, so we only have to prove that for any $v,\langle v, v\rangle<0$, then $R_{v_{i}}(v)$ and $v$ are in the same component of the interior of the light cone. Suppose

$$
\begin{gathered}
v=v^{\prime}+a q, \quad a>0, \quad\left\langle q, v^{\prime}\right\rangle=0, \\
v_{i}=u_{i}+q, \quad\left\langle q, u_{i}\right\rangle=0,
\end{gathered}
$$

then

$$
\begin{aligned}
\left\langle R_{v_{i}}(v), q\right\rangle & =-a-2 \frac{\left\langle v_{i}, v\right\rangle}{\left\langle v_{i}, v_{i}\right\rangle}\left\langle v_{i}, q\right\rangle=-a+2 \frac{\left\langle u_{i}, v^{\prime}\right\rangle-a}{\left\langle u_{i}, u_{i}\right\rangle-1} \\
& <-a+\frac{2 a\left|u_{i}\right|}{\left\langle u_{i}, u_{i}\right\rangle-1} \text { since }\left|v^{\prime}\right|<a \\
& <0 .
\end{aligned}
$$

So, $R_{v_{i}}(v), v$ are in the same component of the interior of the light cone.

Hence $\left\langle v_{i}, u\right\rangle=0$, and $u \neq 0$. Contradiction.

Therefore $M$ has one and only one curvature normal with negative length. 
Since $R_{v_{i}}^{q}\left(v_{1}\right)=c v_{\sigma_{i}(1)}$, where $c \in R$, and $R_{v_{i}}^{q} \in O\left(\nu(M)_{q}\right)$, so we have $\sigma_{i}(1)=1$ and $c= \pm 1$.

Since $R_{v_{i}}^{q}\left(v_{1}\right), v_{1}$ are in the same component of the interior of the light cone, $c=1$. i.e.,

$$
R_{v_{i}}^{q}\left(v_{1}\right)=v_{1} \quad \text { for } i=2, \ldots, p .
$$

So, $\left\langle v_{1}, v_{i}\right\rangle=0$ for $i=2, \ldots, p$.

2.5 Corollary. Suppose $M^{n} \subset H^{n+k}$ is a full isoparametric submanifold, $v_{1}, \ldots, v_{p}$ are the curvature normals, where $\left\langle v_{1}, v_{1}\right\rangle<0,\left\langle v_{i}, v_{i}\right\rangle>0$ if $i>1$, $E_{i}$ the corresponding curvature distributions.

If $e_{j} \in E_{1}, e_{k} \in E_{i}, i>1$, then $\omega_{j k}=0$.

Proof. Let $\omega_{j k}=\sum_{l} \gamma_{j k}^{l} \omega_{l}$. By (1.6), we know that if $e_{l} \in E_{1}$, or $E_{i}$, then $\gamma_{j k}^{l}=0$.

Suppose $\gamma_{j k}^{l} \neq 0$ and $e_{l} \in E_{s}, s \neq 1, i$, then

$$
v_{s}=\left(1-a_{1 i}^{s}\right) v_{1}+a_{1 i}^{s} v_{i} \text { by }(1.6) \text {. }
$$

By (2.4) we have $\left\langle v_{1}, v_{s}\right\rangle=0,\left\langle v_{1}, v_{i}\right\rangle=0$. So, $a_{1 i}^{s}=1, v_{s}=v_{i}$, a contradiction. Hence we have $\gamma_{j k}^{l}=0$, and therefore

$$
\omega_{j k}=\sum_{l} \gamma_{j k}^{l} \omega_{l}=0
$$

2.6 Corollary. Suppose $M^{n} \subset H^{n+k} \subset R^{n+k, 1}$ is a full isoparametric submanifold, $v_{1}, v_{2}, \ldots, v_{p}$ curvature normals, such that $\left|v_{1}\right|<0,\left|v_{i}\right|>0$ for $i>1$, $E_{1}, E_{2}, \ldots, E_{p}$ are the corresponding curvature distributions and $\operatorname{dim} E_{1}=m$.

Then there exists a Lorentz subspace $V^{m+1}$ of $R^{n+k, 1}$, such that

$$
M=H^{m}\left(\frac{1}{\left\langle v_{1}, v_{1}\right\rangle}\right) \times M_{1}^{n-m}
$$

where

$$
H^{m}\left(\frac{1}{\left\langle v_{1}, v_{1}\right\rangle}\right)=\left\{x \in V^{m+1} \mid\langle x, x\rangle=\frac{1}{\left\langle v_{1}, v_{1}\right\rangle}\right\}
$$

and

$$
M_{1}^{n-m} \subset S^{n+k-m-1}\left(-1-\frac{1}{\left\langle v_{1}, v_{1}\right\rangle}\right) \subset V^{\perp}
$$

is an isoparametric submanifold.

Proof. Let

$$
X^{*}=X+\frac{v_{1}}{\left\langle v_{1}, v_{1}\right\rangle}: M \rightarrow R^{n+k, 1}
$$

Then

$$
\mathrm{d} X^{*}=\bigoplus_{i=1}^{p}\left(1-\frac{\left\langle v_{1}, v_{i}\right\rangle}{\left\langle v_{1}, v_{1}\right\rangle}\right) \mathrm{id}_{E_{i}}=\bigoplus_{i>1} \mathrm{id}_{E_{i}} .
$$

So, $X^{*}(M)=M_{1}$ is a submanifold, and $X^{*}: M \rightarrow M_{1}$ is a Riemannian submersion, $E_{1}$ is the vertical distribution, and $\bigoplus_{i>1} E_{i}$ is the horizontal distribution. 
From (2.5), we have

$$
\omega_{j k}=0 \text { if } e_{j} \in E_{1}, e_{k} \in \bigoplus_{i>1} E_{i}
$$

So, the O'Neil tensor $A\left[\mathrm{O}^{\prime} \mathrm{N} 1\right]$ is zero, therefore the horizontal distribution $\bigoplus_{i>1} E_{i}$ is integrable. Let $N(q)$ denote the leaf $\bigoplus_{i>1} E_{i}$ through $q, q \in M$.

Claim. $E_{1}(x) \oplus R v_{1}(x)$ is a fixed $m+1$ plane in $R^{n+k, 1}$ for $x$ in $N(q)$.

Proof of the claim. If $e_{j} \in E_{i}, i>1$, then $\nabla_{e_{j}} v_{1}=-\left\langle v_{1}, v_{i}\right\rangle e_{j}=0$. So, $e_{1} \wedge \cdots \wedge e_{m} \wedge \mathrm{d} v_{1}=0$.

Suppose $e_{1}, \ldots, e_{m} \in E_{1}$, then

$$
\begin{aligned}
\mathrm{d}\left(e_{1}\right. & \left.\wedge \cdots \wedge e_{m} \wedge v_{1}\right) \\
& =\sum_{j=1}^{m} e_{1} \wedge \cdots \wedge \mathrm{d} e_{j} \wedge \cdots \wedge e_{m} \wedge v_{1}+e_{1} \wedge \cdots \wedge e_{m} \wedge \mathrm{d} v_{1} \\
& =\sum_{j=1}^{m} e_{1} \wedge \cdots \wedge\left(\sum_{k>m} \omega_{j k} e_{k}+\sum_{\alpha} \omega_{j k} e_{\alpha}\right) \wedge \cdots \wedge e_{m} \wedge v_{1} \\
& =\sum_{j=1}^{m} e_{1} \wedge \cdots \wedge\left(\sum_{\alpha} \omega_{j \alpha} e_{\alpha}\right) \wedge \cdots \wedge e_{m} \wedge v_{1} \quad \text { by }(2.5) \\
& =\sum_{j=1}^{m} e_{1} \wedge \cdots \wedge\left(\sum_{\alpha \leq n+k}\left\langle v_{1}, e_{\alpha}\right\rangle e_{\alpha}+e_{n+k+1}\right) \wedge \cdots \wedge e_{m} \wedge v_{1} \\
& =\sum_{j=1}^{m} e_{1} \wedge \cdots \wedge v_{1} \wedge e_{j+1} \wedge \cdots \wedge e_{m} \wedge v_{1} \\
& =0
\end{aligned}
$$

So, $E_{1}(x) \oplus R v_{1}(x)$ is constant for $x$ in $N(q)$.

From (1.11) we know that $E_{1}(x) \oplus R v_{1}(x)$ is also fixed on any fiber of $X^{*}$, so $V^{m+1}=E_{1}(x) \oplus R v_{1}(x)$ is a fixed $m+1$ Lorentz subspace of $R^{n+k, 1}$.

Since

$$
\left\langle x+\frac{v_{1}(x)}{\left\langle v_{1}, v_{1}\right\rangle}, v_{1}(x)\right\rangle=0, \quad X^{*}(x)=x+\frac{v_{1}(x)}{\left\langle v_{1}, v_{1}\right\rangle} \in V^{\perp}
$$

for any $x \in M$, i.e., $X^{*}(M)=M_{1} \subset V^{\perp}$. Moreover, by direct computation we see that $X^{*}(M)=M_{1}$ is isoparametric in the sphere of $V^{\perp}$ with radius $\left(-1-1 /\left\langle v_{1}, v_{1}\right\rangle\right)^{1 / 2}$, centered at the origin.

Suppose $P$ is the orthogonal projection of $R^{n+k, 1}$ onto $V$. Then

$$
P\left|M: M \rightarrow H^{m}\left(\frac{1}{\left\langle v_{1}, v_{1}\right\rangle}\right), \quad P\right| M(x)=-\frac{v_{1}(x)}{\left\langle v_{1}, v_{1}\right\rangle}
$$

is surjective, and $X^{*}=1-P \mid M$. Therefore $M=H^{m} \times M_{1}$.

\section{Polar actions on $H^{n}$}

Suppose $G$ is a connected, closed Lie subgroup of $O(n, 1)$, and the isometric action of $G$ on $H^{n}$ is polar (see [PT1]), i.e., $H^{n}$ has a connected, 
closed submanifold which meets all orbits orthogonally. Any such submanifold is called a section of $H^{n}$. Suppose $q \in H^{n}$ is a regular point, $H\left(\Sigma_{q}\right)$ is the unique section through $q$. Since sections are totally geodesic submanifolds of $H^{n}$, therefore $H\left(\Sigma_{q}\right)=\Sigma_{q} \cap H^{n}$, where $\Sigma_{q}$ is a $(m, 1)$ Lorentz subspace of $R^{n, 1}$.

3.1 Theorem. Suppose $q \in H^{n}$ is a regular point and $G q \subset l$, where $l$ is a hyperplane of $R^{n, 1}$. Then

$$
G\left(H\left(\Sigma_{q}\right) \cap l\right)=l \cap H^{n},
$$

in particular $G\left(l \cap H^{n}\right) \subset l \cap H^{n}$. Moreover, the action of $G$ on $l \cap H^{n}$ is polar. Proof. Suppose $v$ is a normal vector of $l$, where $l=\left\{x \in R^{n, 1} \mid\langle x, v\rangle=c\right\}$. Then $v \in \nu_{g q}(G q)$, the normal space of $G q$ in $R^{n, 1}$ at $g q$, for all $g \in G$.

(1) If $x \in H\left(\Sigma_{q}\right) \cap l$, then $\langle\xi g x, v\rangle=0$ for all $g \in G, \xi \in \mathbf{g}$, the Lie algebra of $G$, hence, $\langle g x, v\rangle$ is constant on $G$. Therefore, we have

$$
\langle g x, v\rangle=\langle x, v\rangle=c \quad \forall g \in G,
$$

i.e., $G x \subset l$. So, $G\left(H\left(\Sigma_{q}\right) \cap l\right) \subset l \cap H^{n}$.

(2) Since $H^{n}=G H\left(\Sigma_{q}\right)$, for any $y \in H^{n} \cap l$ there exist $h \in G, x \in H\left(\Sigma_{q}\right)$, such that $y=h x$. Because $\langle g x, v\rangle$ is constant on $G$ we have $\langle x, v\rangle=$ $\langle h x, v\rangle=\langle y, v\rangle=c$, i.e., $x \in l$. So, $G\left(H\left(\Sigma_{q}\right) \cap l\right) \supset l \cap H^{n}$.

From (1) and (2) we have

$$
G\left(H\left(\Sigma_{q}\right) \cap l\right)=l \cap H^{n},
$$

so $G$ acts on $l \cap H^{n}$ and the action is polar.

3.2. Suppose some principal orbit of $G$ is not included in any hyperplane. Then all principal orbits of $G$ in $H^{n}$ are full isoparametric submanifolds of $H^{n}$.

3.3 Theorem. Suppose $G q$ is a principal orbit of $G, q \in H^{n}, v_{1}$ is the curvature normal of $G q$ in $R^{n, 1}$ such that $\left\langle v_{1}, v_{1}\right\rangle<0, E_{1}$ is the corresponding curvature distribution, $V=E_{1}(q) \oplus R v_{1}(q), \operatorname{dim}\left(E_{1}\right)=m$. Then

(1) $V$ is $G$-invariant $(m, 1)$ subspace of $R^{n, 1}$, and

$$
\rho: G \rightarrow S O(V), \quad \rho^{\perp}: G \rightarrow S O\left(V^{\perp}\right)
$$

are polar representations.

(2) There exists a polar representation $G_{1} \subset S O\left(V^{\perp}\right)$ such that the orbits of $G$ in $H^{n}$ coincide with the orbits

$\left\{S O(m, 1) u_{1} \times G_{1} u_{2} \mid u_{1} \in V, u_{2} \in V^{\perp},\left\langle u_{1}, u_{1}\right\rangle<-1\right.$, and $\left.\left|u_{1}\right|^{2}+\left|u_{2}\right|^{2}=-1\right\}$.

Proof. (1) From the proof of (2.6) we know,

$$
V(g q)=E_{1}(g q) \oplus R v_{1}(g q)=E_{1}(q) \oplus R v_{1}(q)=V .
$$

So $g V=V$ for all $g \in G$, i.e., $V$ is invariant under $G$. Moreover $\rho, \rho^{\perp}$ are clearly polar.

(2) Let $q_{1}=-v_{1}(q) /\left|v_{1}\right| \in H^{n}$. Then from (2.6) we know

$$
G q_{1}=\{x \in V \mid\langle x, x\rangle=-1\},
$$

so $V=T_{q_{1}} G q_{1} \oplus R q_{1}$, hence the normal space of $G q_{1}$ at $q_{1}$ in $H^{n}$ is $V^{\perp}$. 
Let $G_{1}$ be the identity component of $G_{q_{1}}$. Then $G_{1} \subset O\left(V^{\perp}\right)$ is a slice representation of $H^{n}$, so it is a polar representation and $T_{q_{1}} H\left(\Sigma_{q}\right)$ is a section of $G_{1}$.

Let $q_{2}=-v_{1}(q) /\left\langle v_{1}, v_{1}\right\rangle, q_{3}=q+v_{1}(q) /\left\langle v_{1}, v_{1}\right\rangle$. Then $q=q_{2}+q_{3}$, and $q_{2} \in V, q_{3} \in V^{\perp}$. Since $q$ is a regular point of $G$, so $q_{3}$ is a regular point of $\rho^{\perp}: G \rightarrow S O\left(V^{\perp}\right)$.

Denote the identity component of $G_{q_{3}}$ by $K$. Then $K$ fixes every vector in $T_{q_{1}} H\left(\Sigma_{q}\right) \subset V^{\perp}$. Since

$$
G q=G q_{2} \times G q_{3},
$$

so

$$
G_{1} q_{3}=G q_{3}, \quad K q_{2}=G q_{2} .
$$

Suppose $\mathbf{g}, \mathbf{g}_{1}, \mathbf{k}$ are the Lie algebras of $G, G_{1}, K$ respectively. Then $\mathbf{g}_{1} q_{3}=$ $\mathbf{g} q_{3}, \mathbf{k} q_{2}=\mathbf{g} q_{2}$.

Claim. $\mathbf{g}=\mathbf{k}+\mathbf{g}_{1}$ and $G=G_{1} K=K G_{1}$.

Proof of the claim.

$$
\mathbf{g} q=\mathbf{g} q_{2}+\mathbf{g} q_{3}=\mathbf{k} q_{2}+\mathbf{g}_{1} q_{3}=\left(\mathbf{k}+\mathbf{g}_{1}\right) q_{2}+\left(\mathbf{k}+\mathbf{g}_{1}\right) q_{3}=\left(\mathbf{k}+\mathbf{g}_{1}\right) q,
$$

and if $X q=0, X \in \mathbf{g}$, then $X q_{2}=X q_{3}=0$, so $X \in \mathbf{k} \cap \mathbf{g}_{1}$, hence $\mathbf{g}=\mathbf{k}+\mathbf{g}_{1}$ and $G=G_{1} K=K G_{1}$.

Now consider the $K \times G_{1}$ action on $V \oplus V^{\perp}$. For any $u_{1}+u_{2} \in H\left(\Sigma_{q}\right)$, where $u_{1} \in R v_{1}, u_{2} \in T_{q_{1}} H\left(\Sigma_{q}\right)$,

$$
G\left(u_{1}+u_{2}\right) \subset K u_{1} \times G_{1} u_{2},
$$

and, because $\mathbf{g}=\mathbf{k}+\mathbf{g}_{1}$, by dimension and connectedness we have

$$
G\left(u_{1}+u_{2}\right)=K u_{1} \times G_{1} u_{2} .
$$

By (2.6) we know

$$
K \frac{v_{1}(q)}{\left\langle v_{1}, v_{1}\right\rangle}=\left\{x \in V \mid\langle x, x\rangle=\frac{1}{\left\langle v_{1}, v_{1}\right\rangle}\right\},
$$

so, the orbits of $G$ in $H^{n}$ coincide with the orbits

$$
\begin{array}{r}
\left\{S O(m, 1) u_{1} \times G_{1} u_{2} \mid u_{1} \in V, u_{2} \in V^{\perp},\left\langle u_{1}, u_{1}\right\rangle<-1,\right. \\
\text { and } \left.\left|u_{1}\right|^{2}+\left|u_{2}\right|^{2}=-1\right\} .
\end{array}
$$

\section{ISOPARAMETRIC SUBMANIFOLD OF}

RIEMANNIAN HILBERT HYPERBOLIC SPACE

Suppose $V$ is a separable Hilbert space. Let $\widetilde{V}=R \oplus V$ with the inner product

$$
\langle(s, x),(t, y)\rangle=-s t+\langle x, y\rangle \quad \text { where } s, t \in R, x, y \in V .
$$

Then $\widetilde{V}$ is a Lorentz Hilbert space. Let $H(\widetilde{V})=\left\{(s, x) \in \widetilde{V} \mid-s^{2}+\langle x, x\rangle=\right.$ $-1, s>0\}$, then $H(\tilde{V})$ is a Riemannian Hilbert hyperbolic space with constant sectional curvature -1 . 
4.1 Definition. A Hilbert submanifold $M$ of $H(\widetilde{V})$ is called isoparametric if $M$ satisfies the following conditions:

(1) $\operatorname{Codim}(M)<\infty$.

(2) $\nu(M)$, the normal bundle of $M$ in $H(\widetilde{V})$, is globally flat.

(3) If $v$ is a parallel normal vector field on $M$, then $A_{v(x)}, A_{v(y)}$ are orthogonally equivalent for all $x, y \in M$.

(4) $A_{v}$ is compact for any $v \in \nu(M)_{x}$.

4.2 Proposition. Suppose $M$ is not a full isoparametric Hilbert submanifold of $H(\widetilde{V})$, i.e., there is a nonzero vector $v$ in $\widetilde{V}$, such that $\langle x, v\rangle$ is a constant on $M$, then $\langle v, v\rangle>0$, and $\langle x, v\rangle=0$ on $M$.

Proof. Suppose $X: M \subset H(\widetilde{V}) \subset \widetilde{V}$, then we have $\langle X, v\rangle=a,\langle\mathrm{~d} X, v\rangle=0$. So, there exists a normal vector field $Z$ of $M$ in $H(\widetilde{V})$, such that $v=a X+b Z$, where $b \neq 0$. Hence we have

$$
0=a \mathrm{~d} X+b \mathrm{~d} Z, \quad A_{Z}=\frac{a}{b} \mathrm{id}_{T M} .
$$

Since $A_{Z}$ is a compact operator, $a=0$.

Therefore $\langle v, v\rangle>0$ and $\langle x, v\rangle=0$ for all $x \in M$.

4.3 Theorem. If $M \subset H(\tilde{V})$ is a full isoparametric submanifold, then $\operatorname{dim}(M)$ $<\infty$.

In order to prove Theorem 4.3, we need the following lemmas, in which we will always assume that $M$ is a full isoparametric in $H(\widetilde{V})$ and $\nu(M)$ is the normal bundle of $M$ in $H(\widetilde{V})$.

4.4 Lemma. There exist smooth distributions $E_{i}, i \in I$, and smooth parallel normal fields $v_{i} \in \nu(M), i \in I$, such that

$$
T M=\bigoplus_{i \in I} E_{i}
$$

and for any $v \in \nu(M)$,

$$
A_{v} \mid E_{i}=\left\langle v, v_{i}\right\rangle \mathbf{i d}_{E_{i}} .
$$

Moreover, if we suppose $v_{0}=0$, then $\operatorname{dim}\left(E_{i}\right)<\infty$, for $i \neq 0$.

Proof. The same proof as for the finite dimension case works here, because $A_{v}$ is compact and selfadjoint.

$E_{i}$ are called curvature distributions, $v_{i}$ are called curvature normals. We will assume that $\left\{e_{i} \mid i \in N\right\}$ is an orthonormal frame for $H(\widetilde{V})$, such that $\left\{e_{i} \mid i>k\right\}$ is an orthonormal tangent frame for $M$, where $e_{i}$ is in some curvature distribution, and $\left\{e_{\alpha} \mid 1 \leq \alpha \leq k\right\}$ is an orthogonal normal frame of $M$ in $H(\widetilde{V})$.

4.5 Lemma. $M$ is full in $H(\tilde{V})$ iff $v_{i}, i \in I$, span $\nu(M)$.

Proof. The same proof as for (1.4).

4.6 Lemma. For $\varepsilon>0, I_{\varepsilon}=\left\{\left.i \in I|| v_{i}\right|^{2}>\varepsilon\right\}$ is finite.

Proof. Since $A_{e_{\alpha}}$ is compact

$$
\left\{i \in I||\left\langle v_{i}, e_{\alpha}\right\rangle \mid>\sqrt{\frac{\varepsilon}{k}}\right\} \text { is finite, }
$$


hence

$$
I_{\varepsilon} \subset \bigcup_{1 \leq \alpha \leq k}\left\{i \in I||\left\langle v_{i}, e_{\alpha}\right\rangle \mid>\sqrt{\frac{\varepsilon}{k}}\right\} \quad \text { is finite. }
$$

4.7 Lemma. Let $\omega_{i j}=\sum_{l} \gamma_{i j}^{l} \omega_{l}$. Then

$$
\gamma_{i j}^{l}\left(v_{i^{\prime}}-v_{j^{\prime}}\right)=\gamma_{i l}^{j}\left(v_{i^{\prime}}-v_{l^{\prime}}\right)=\gamma_{l j}^{i}\left(v_{l^{\prime}}-v_{j^{\prime}}\right), \quad j \neq l,
$$

where $e_{i} \in E_{i^{\prime}}, e_{j} \in E_{j^{\prime}}, \varepsilon_{l} \in E_{l^{\prime}}$.

Proof. The same proof as for (1.5).

4.8 Corollary. Suppose $e_{i} \in E_{i^{\prime}}, e_{j} \in E_{j^{\prime}}, i^{\prime} \neq j^{\prime}$, then

(1) $\gamma_{i j}^{l}=0$ if $e_{l} \in E_{j^{\prime}}$.

(2) If $\gamma_{i j}^{l} \neq 0$, where $e_{l} \in E_{l^{\prime}}, l^{\prime} \neq j^{\prime}$, then

$$
v_{l^{\prime}}=\left(1-a_{i^{\prime} j^{\prime}}^{l^{\prime}}\right) v_{i^{\prime}}+a_{i^{\prime} j^{\prime}}^{l^{\prime}} v_{j^{\prime}}, \quad \text { where } a_{i^{\prime} j^{\prime}}^{l^{\prime}} \neq 0,1
$$

and

$$
\gamma_{i j}^{l}=\gamma_{i l}^{j} a_{i^{\prime} j^{\prime}}^{l^{\prime}}=\gamma_{l j}^{i}\left(1-a_{i^{\prime} j^{\prime}}^{l^{\prime}}\right) .
$$

4.9 Lemma. Suppose $v_{i} \neq v_{j}$, then

$$
\left\langle v_{i}, v_{j}\right\rangle-1=\sum_{\substack{l \\ v_{l}=\left(1-a_{i j}^{l}\right) v_{i}+a_{i j}^{l} v_{j} \\ a_{i j}^{l} \neq 0,1}} \sum_{\substack{t \\ e_{l} \in E_{l}}}\left(\gamma_{i j}^{t}\right)^{2} \frac{1}{a_{i j}^{l}\left(a_{i j}^{l}-1\right)} .
$$

Proof. The same proof as for (2.1).

4.10 Lemma. (1) $M$ has at most one curvature normal whose length $\leq 1$.

(2) If $v_{i}$ is a curvature normal of $M$, then $v_{i} \neq 0$.

Proof. (1) Suppose there are at least two curvature normals whose length $\leq 1$. Then, by (4.6), we can choose $v_{i}, v_{j}, i \neq j$, such that $\left|v_{i}\right| \leq 1,\left|v_{j}\right| \leq 1$, and if $\left|v_{l}\right| \leq 1$, then $\left|v_{i}\right| \geq\left|v_{l}\right|$ and $\left\langle v_{i}, v_{j}\right\rangle \geq\left\langle v_{i}, v_{l}\right\rangle$, for $l \neq i$.

By (4.9)

$$
\left\langle v_{i}, v_{j}\right\rangle-1=\sum_{\substack{l \\ v_{l}=\left(1-a_{i j}^{l}\right) v_{i}+a_{i j}^{l} v_{j} \\ a_{i j}^{l} \neq 0,1}} \sum_{\substack{t \\ e_{t} \in E_{l}}}\left(\gamma_{i j}^{t}\right)^{2} \frac{1}{a_{i j}^{l}\left(a_{i j}^{l}-1\right)}
$$

and $\left\langle v_{i}, v_{j}\right\rangle<\left\langle v_{i}, v_{i}\right\rangle \leq 1$, we have

$$
\sum_{\substack{l \\ v_{l}=\left(1-a_{i j}^{l}\right) v_{i}+a_{i j}^{l} v_{j} \\ a_{i j}^{l} \neq 0,1}} \sum_{\substack{t \\ e_{t} \in E_{l}}}\left(\gamma_{i j}^{t}\right)^{2} \frac{1}{a_{i j}^{l}\left(a_{i j}^{l}-1\right)}<0 .
$$

On the other hand, if $\left|v_{l}\right| \leq 1$, and $v_{l}=a_{i j}^{l} v_{i}+\left(1-a_{i j}^{l}\right) v_{j}, 0<a_{i j}^{l}<1$, then

$$
\begin{aligned}
\left\langle v_{i}, v_{l}\right\rangle & =a_{i j}^{l}\left\langle v_{i}, v_{i}\right\rangle+\left(1-a_{i j}^{l}\right)\left\langle v_{i}, v_{j}\right\rangle \\
& >a_{i j}^{l}\left\langle v_{i}, v_{j}\right\rangle+\left(1-a_{i j}^{l}\right)\left\langle v_{i}, v_{j}\right\rangle \\
& =\left\langle v_{i}, v_{j}\right\rangle,
\end{aligned}
$$


a contradiction. So, if $\left|v_{l}\right| \leq 1$, and $v_{l}=a_{i j}^{l} v_{i}+\left(1-a_{i j}^{l}\right) v_{j}$, then, either $a_{i j}^{l}<0$, or $a_{i j}^{l}>1$.

If $\left|v_{l}\right|>1$, and $v_{l}=a_{i j}^{l} v_{i}+\left(1-a_{i j}^{l}\right) v_{j}$, then, either $a_{i j}^{l}<0$, or $a_{i j}^{l}>1$. So, we have

$$
\sum_{\substack{l \\ v_{l}=\left(1-a_{i j}^{l}\right) v_{i}+a_{i j}^{l} v_{j} \\ a_{i j}^{l} \neq 0,1}} \sum_{\substack{t \\ e_{t} \in E_{l}}}\left(\gamma_{i j}^{t}\right)^{2} \frac{1}{a_{i j}^{l}\left(a_{i j}^{l}-1\right)} \geq 0,
$$

a contradiction. Thus $M$ has at most one curvature normal whose length $\leq 1$.

(2) Suppose $v_{0}=0$ is a curvature normal of $M$. By (1) and (4.6) we know that $M$ has only finitely many curvature normals, and $M$ has at least one nonzero curvature normal, since $M$ is full. Hence we can choose $v_{j}$, such that $\left|v_{j}\right| \leq\left|v_{l}\right|$, for all $l \neq 0$. Then, by (4.9) we have

$$
\begin{aligned}
\left\langle v_{0}, v_{j}\right\rangle-1= & \sum_{\substack{l \\
v_{l}=\left(l-a_{i j}^{l}\right) v_{0}+a_{i j}^{l} v_{j} \\
a_{i j}^{l} \neq 0,1}} \sum_{\substack{t \\
e_{t} \in E_{l}}}\left(\gamma_{i j}^{t}\right)^{2} \frac{1}{a_{i j}^{l}\left(a_{i j}^{l}-1\right)}-1 \\
= & \sum_{\substack{l \\
v_{l}=a_{i j}^{l} v_{j} e_{t} \in E_{l} \\
a_{i j}^{l} \neq 0,1}} \sum_{\substack{t \\
e_{i j}}}\left(\gamma^{t}\right)^{2} \frac{1}{a_{i j}^{l}\left(a_{i j}^{l}-1\right)} .
\end{aligned}
$$

On the other hand, if $0<a_{i j}^{l}<1$, and $v_{l}=a_{i j}^{l} v_{j}$, then $\left|v_{l}\right|=a_{i j}^{l}\left|v_{j}\right|<\left|v_{j}\right|$, a contradiction. So, if $v_{l}=a_{i j}^{l} v_{j}$, then either $a_{i j}^{l}<0$, or $a_{i j}^{l}>1$. Hence we have

$$
\sum_{\substack{l \\ v_{l}=a_{i j}^{l} v_{j} \\ a_{i j}^{l} \neq 0,1}} \sum_{\substack{t \\ e_{t} \in E_{l}}}\left(\gamma_{i j}^{t}\right)^{2} \frac{1}{a_{i j}^{l}\left(a_{i j}^{l}-1\right)} \geq 0,
$$

a contradiction. Therefore $M$ has no zero curvature normal.

4.11 Proof of Theorem 4.3. By (4.6) and (4.10), we know that $M$ has only a finite number of curvature normals and none of them is zero. Therefore, by (4.4), $M$ is of finite dimension.

4.12 Theorem. Suppose $M$ is an isoparametric Hilbert submanifold of $H(\tilde{V})$, then $M$ is either of finite dimension, or a totally geodesic submanifold of $H(\widetilde{V})$, i.e., $M=H(\widetilde{V}) \cap V_{1}$, where $V_{1}$ is a Lorentz Hilbert subspace of $\widetilde{V}$.

Proof. The direct corollary of (4.2) and (4.3).

\section{REFERENCES}

[Ab] U. Abresch, Isoparametric hypersurfaces with four or six distinct principal curvatures, Math. Ann. 264 (1983), 283-302.

[Be] C. T. Benson and L. C. Grove, Finite reflection groups, Bogden \& Quigley, 1971.

[Ca1] E. Cartan, Familles de surface isoparametrique dans les space a courbure constante, Ann. of Math. (2) 17 (1938), 177-191. 
[Ca2] _- Sur des familles remarquables d'hypersurfaces isoparametriques dans les espaces spheriques, Math. Z. 45 (1939), 335-367.

[Ca3] __, Sur quelques familles remarquables d'hypersurfaces, C. R. Congres. Math. Liège, 1939, pp. 30-41.

[Ca4] __ Sur des familles d'hypersurfaces isoparametriques des espaces spheriques a 5 et a 9 dimension, Univ. Nac. Tucuman Rev. Ser. A 1 (1940), 5-22.

[Co1] L. Conlon, A class of variationally complete representations, J. Differential Geom. 7 (1972), 149-160.

[Co2] _ Variational completeness and K-transversal domains, J. Differential Geom. 7 (1972), 135-147.

[CR] T. E. Cecil and P. J. Ryan, Tight and taut immersions of manifolds, Research Notes in Math., vol. 107, Pitman, Boston, Mass., 1985.

[CW1] S. Carter and A. West, Generalized Cartan polynomials, J. London Math. Soc. (2) 32 (1984), 305-316.

[CW2] _ Isoparametric systems and transnormality, Proc. London Math. Soc. (3) 51 (1985), 520-542.

[CW3] _ Isoparametric and totally focal submanifolds, Preprint, University of Leeds, 1988.

[Da] J. Dadok, Polar coordinates induced by actions of compact Lie groups, Trans. Amer. Math. Soc. 288 (1985), 479-502.

[FKM] D. Ferus, H. Kacher and H. F. Münzner, Cliffordalgebren and neue isoparametische hyperflächen, Math. Z. 177 (1981), 479-502.

[Ha] C. E. Harle, Isoparametric families of submanifolds, Bol. Soc. Brasil. Mat. 13 (1982), 35-48.

[HL] W. Y. Hsiang and B. H. Lawson, Jr., Minimal submanifolds of low cohomogeneity, J. Differential Geom. 5 (1971), 1-38.

[HPT] W. Y. Hsiang, R. S. Palais and C. L. Terng, The topology of isoparametric submanifolds, J. Differential Geom. 27 (1988), 423-460.

[La] S. Lang, Introduction to differentiable manifolds, Interscience, New York, 1966.

[Mü] H. F. Münzner, Isoparametrische hyperflächen in spharen. I, II, Math. Ann. 251 (1980), 57-71; 256 (1981), 215-232.

[No] K. Nomizu, Elie Cartan's work on isoparametric families of hypersurfaces, Proc. Sympos. Pure Math., vol. 27, Amer. Math. Soc., Providence, R.I., 1975, pp. 191-200.

[O'N1] B. O'Neill, The fundamental equations of a submersion, Michigan Math. J. 13 (1966), 459469.

[O'N2] __ Semi-Riemannian geometry with applications to relativity, Academic Press, 1983.

[OT] H. Ozeki and M. Takeuchi, On some types of isoparametric hypersurfaces in spheres. I, II, Tohoku Math. J. 27 (1975), 515-559 and 28 (1976), 7-55.

[PT1] R. S. Palais and C. L. Terng, A general canonical form theory, Trans. Amer. Math. Soc. 300 (1987), 771-789.

[PT2] _ Critical point theory and submanifold geometry, Lecture Notes in Math., vol. 1353, Springer-Verlag, 1988.

[Te1] C. L. Terng, Isoparametric submanifolds and their Coxeter groups, J. Differential Geom. 21 (1985), 79-107.

[Te2] _ Convexity theorem for isoparametric submanifolds, Invent. Math. 85 (1986), 487-492.

[Te3] _ _ Submanifolds with flat normal bundle, Math. Ann. 277 (1987), 95-111.

[Te4] __, Proper Fredholm submanifolds of Hilbert space, J. Differential Geom. 29 (1989), 9-47.

[Th] G. Thorbergsson, Isoparametric foliations and their buildings, preprint.

Department of Mathematics, Brandeis University, Waltham, Massachusetts 02254 Current address: Department of Mathematics, University of Pennsylvania, Philadelphia, Pennsylvania 19104-6395

E-mail address: wu@math.upenn.edu 